University of Nebraska - Lincoln

DigitalCommons@University of Nebraska - Lincoln

2012

\title{
Benchmark assessment of automated delamination propagation capabilities in finite element codes for static loading
}

Adrian C. Orifici

RMIT University

Ronald Krueger

National Institute of Aerospace (NIA)

Follow this and additional works at: https://digitalcommons.unl.edu/nasapub

Part of the Physical Sciences and Mathematics Commons

Orifici, Adrian C. and Krueger, Ronald, "Benchmark assessment of automated delamination propagation capabilities in finite element codes for static loading" (2012). NASA Publications. 75.

https://digitalcommons.unl.edu/nasapub/75

This Article is brought to you for free and open access by the National Aeronautics and Space Administration at DigitalCommons@University of Nebraska - Lincoln. It has been accepted for inclusion in NASA Publications by an authorized administrator of DigitalCommons@University of Nebraska - Lincoln. 


\title{
Benchmark assessment of automated delamination propagation capabilities in finite element codes for static loading
}

\author{
Adrian C. Orifici ${ }^{\mathrm{a}, *}$, Ronald Krueger ${ }^{\mathrm{b}}$ \\ ${ }^{a}$ RMIT University, GPO Box 2476, Melbourne, Victoria 3001, Australia

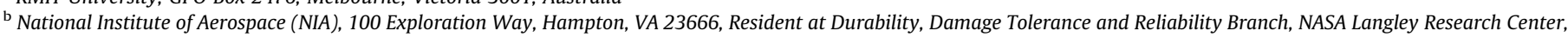 \\ MS 188E, Hampton, VA 23681, USA
}

\section{A R T I C L E I N F O}

\section{Article history:}

Received 10 December 2010

Received in revised form

20 September 2011

Accepted 16 January 2012

Available online 8 February 2012

Keywords:

Laminated composite materials

Delamination

Fracture mechanics

Benchmarking

\begin{abstract}
A B S T R A C T
With the increasing implementation into commercial finite element (FE) codes of capabilities for simulating delamination propagation in composite materials, the need for benchmarking and assessing these capabilities is critical. In this study, the capabilities of the commercial FE code Marc ${ }^{\mathrm{TM}} 2008 \mathrm{r} 1$ with implementation of the Virtual Crack Closure Technique (VCCT) was assessed. Benchmark delamination propagation results for several specimen configurations were generated using a numerical approach. Specimens were analyzed with three-dimensional and two-dimensional models, and compared with previous analyses using Abaqus ${ }^{\mathbb{B}}$ with the VCCT implemented. The results demonstrated that the VCCT implementation in $\operatorname{Marc}^{\mathrm{TM}}$ was capable of accurately replicating the benchmark delamination growth results. The analyses in Marc ${ }^{\mathrm{TM}}$ were significantly more computationally efficient than previous analyses in Abaqus ${ }^{\mathbb{R}}$. This was due to a lack of convergence issues, and a solution process that maintained the use of large time increments. The results demonstrated the advantages of numerical over experimental and analytical benchmarks, particularly with regards to comparison of capabilities across codes. More broadly, the results illustrated key similarities and differences between two commercial FE codes implementing the same analysis technique, which reinforces the need for rigorous benchmarking and assessment.
\end{abstract}

(c) 2012 Elsevier B.V. All rights reserved.

\section{Introduction}

One of the most common failure modes for laminated composite structures is delamination, or interlaminar cracking. To characterize the onset and propagation of delamination, the use of fracture mechanics has become common practice over the past two decades [1-3]. The strain energy release rate, $G_{T}$, is typically used as a measure of the driving force for delamination growth in composite laminates. Depending upon external loading, $G_{T}$ can be any combination of its three components, $G_{I}, G_{I I}$, and $G_{I I I}$, which sum to give $G_{T}$. To predict delamination onset or propagation, $G_{T}$ is compared to the interlaminar fracture toughness, $G_{c}$, which is dependent on the relative proportions of the three components. Due to the availability of test methods for characterizing mode I, II and mixed mode I/II delamination, efforts [4-6] have focused on evaluating the dependence of $G_{c}$ on this range of mode mix. Such a quasi-static mixed-mode $\mathrm{I} / \mathrm{II}$ fracture criterion is determined by plotting $G_{C}$ versus the mixed-mode ratio, $G_{I I} / G_{T}$, determined from data generated using pure mode I Double Cantilever Beam (DCB)

\footnotetext{
* Corresponding author. Tel.: +6139925 6092; fax: +61399256108.

E-mail address: adrian.orifici@rmit.edu.au (A.C. Orifici).
}

$\left(G_{I I} / G_{T}=0\right)$, pure mode II End-Notched Flexure (ENF) $\left(G_{I I} / G_{T}=1\right)$ and Mixed-Mode Bending (MMB) tests of varying ratios. This data is used to determine a mathematical relationship between $G_{c}$ and $G_{I I} / G_{T}[7,8]$. An interaction criterion incorporating mode III was recently proposed by Reeder [9].

The virtual crack closure technique (VCCT) is widely used for computing energy release rates, based on results from continuum (2D) and solid (3D) finite element (FE) analyses, and to supply the mode separation required when using mixed-mode fracture criteria [10,11]. The VCCT has been implemented into the commercial FE codes Abaqus ${ }^{\circledR}$ and Marc $^{\mathrm{TM}}$ [12,13], among others, as well as implemented into FE codes using customized approaches for automated crack growth [14,15].

The implementation of any technology into FE codes involves numerical parameters, which can be unique to each code, and need to be understood and calibrated for any analysis. Benchmarking is a straightforward procedure for comparing code capabilities, and judicious selection of appropriate benchmark cases is critical. For delamination growth in composite materials, experimental results used as benchmarks are valuable, but are complicated by aspects such as fiber bridging, crack branching, and experimental variance. Analytical results are also useful as benchmarks, but are not available for all specimen types and 
configurations, and can become complicated and dependent on overly simplifying assumptions.

Numerical benchmarks are attractive, as they allow for a direct assessment of the predictive capabilities of a given code, without being complicated by any of the issues associated with experimental or analytical benchmarks. For assessment of automated delamination propagation capabilities, a numerical benchmark should provide results for delamination growth that are independent of the automation procedure. In this way, the input parameters and performance aspects that are unique to any given software code can be clearly identified and compared across codes.

In response to this, a numerical benchmarking approach was developed in which results for delamination growth are first generated from a series of static analyses with different delamination lengths [16]. These benchmark results are then compared to simulation of delamination propagation using a single analysis. In previous work, this numerical benchmarking approach was applied to assess the implementation of VCCT in Abaqus ${ }^{\mathbb{R}} /$ Standard [16].

In this study, benchmark analyses are performed to assess the automated delamination propagation simulation capabilities of the VCCT implementation in Marc ${ }^{\mathrm{TM}}$ 2008r1. Numerical benchmark delamination growth results are generated for DCB and ENF specimens, following the approach developed previously [16]. These benchmark results are compared to automated delamination propagation analyses from two-dimensional (2D) plane strain and full 3D models. The results are also compared to previous analyses with the VCCT implementation in Abaqus ${ }^{\circledR} /$ Standard. The goals of the current work are to: Demonstrate the application and value of the numerical benchmarking procedure; assess the delamination growth capabilities in Marc ${ }^{\mathrm{TM}}$ for single mode I and II delamination growth specimens; compare the delamination growth capabilities of Marc $^{\mathrm{TM}}$ and Abaqus ${ }^{\circledR}$ for identical specimens.

\section{Specimen description}

For the current investigation, specimens were selected to investigate single mode (I and II) delamination growth with unidirectional laminates. DCB and ENF specimens were chosen, as shown in Figs. 1 and 2, respectively. The DCB specimen was taken from previous work, where numerical benchmark results were generated [16]. The ENF specimen is the three-point bending 3ENF variant, and the specifications were set based on commonality with the DCB specimen. A more extensive study, involving a mixed-mode I/II $\left(G_{I I} / G_{T}=0.4\right)$ Single Leg Bending specimen with a multi-directional laminate and a second DCB specimen with experimental and analytical results, is detailed in

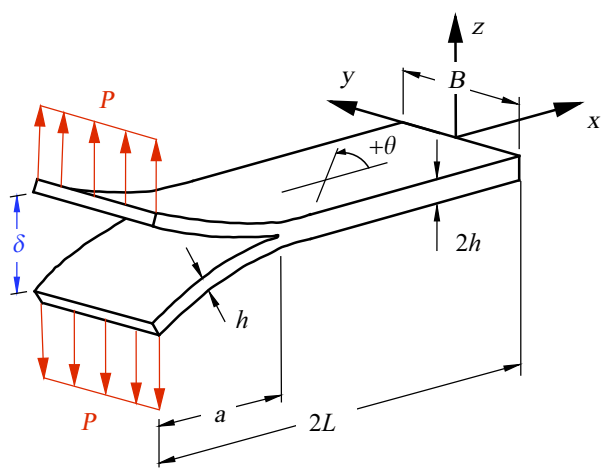

$25.0 \mathrm{~mm}$

$3.0 \mathrm{~mm}$

$150.0 \mathrm{~mm}$

$30.0 \mathrm{~mm}$

T300/1076

unidirectional

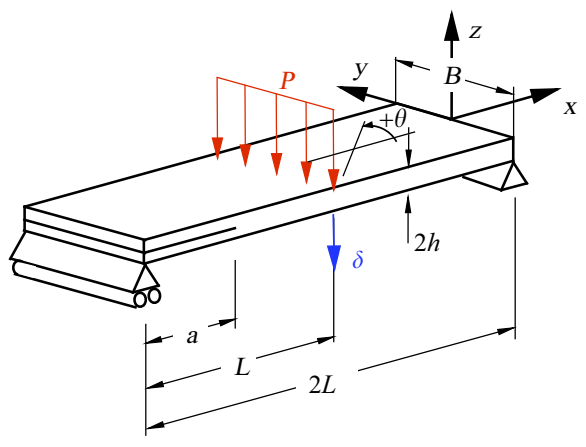

B $\quad 25.0 \mathrm{~mm}$

$\begin{array}{ll}2 h & 3.0 \mathrm{~mm}\end{array}$

L $\quad 70.0 \mathrm{~mm}$

$2 L \quad 140.0 \mathrm{~mm}$

a $\quad 30.0 \mathrm{~mm}$

T300/1076

unidirectional

Fig. 2. End-notched flexure specimen.

Table 1

Material properties.

T300/1076 Unidirectional graphite/Epoxy prepreg

$\begin{array}{lll}E_{11}=139.4 \mathrm{GPa} & E_{22}=10.16 \mathrm{GPa} & E_{33}=10.16 \mathrm{GPa} \\ v_{12}=0.30 & v_{13}=0.30 & v_{23}=0.436 \\ G_{12}=4.6 \mathrm{GPa} & G_{13}=4.6 \mathrm{GPa} & G_{23}=3.54 \mathrm{GPa} \\ G_{I c}=170.3 \mathrm{~J} / \mathrm{m}^{2} & G_{I I c}=493.6 \mathrm{~J} / \mathrm{m}^{2} & G_{I I I c}=493.6 \mathrm{~J} / \mathrm{m}^{2} \\ \eta=1.62 & & \end{array}$

The material properties are given with reference to the ply coordinate axes where index 11 denotes the ply fiber direction, index 22 denotes the direction transverse to the fiber direction in the plane of the lamina and index 33 denotes the direction perpendicular to the plane of the lamina.

Ref. [17]. The specimens used T300/1076 graphite/epoxy with material properties given in Table 1 .

\section{Methodology}

\subsection{Fracture criteria}

Linear elastic fracture mechanics analysis of delamination in composite laminates involves determining the total strain energy release rate, $G_{T}$, and the individual orthogonal components $G_{I}, G_{I I}$ and $G_{I I I}$. The onset of delamination growth is predicted using the failure index:

$\frac{G_{T}}{G_{c}} \geq 1$

The fracture toughness is dependent on the relative proportions of the mode components, or the mode mix. The 3D relationship between $G_{c}$ and modes I, II and III suggested by Reeder [9] is given by

$G_{c}=G_{I c}+\left(G_{I I c}-G_{I c}\right)\left(\frac{G_{I I}+G_{I I I}}{G_{T}}\right)^{\eta}+\left(G_{I I I c}-G_{I I c}\right) \frac{G_{I I I}}{G_{I I}+G_{I I I}}\left(\frac{G_{I I}+G_{I I I}}{G_{T}}\right)^{\eta}$.

where $G_{I c}$ and $G_{I I c}$ are determined experimentally from DCB tests [4] and ENF tests [6], respectively, and the 2D mixed-mode exponent $\eta$ is determined from combining DCB, ENF and MMB tests of varying ratios of $G_{I}$ and $G_{I I}$ [5]. As no standards currently exist for determining $G_{I I I c}$, in this work $G_{\text {IIIC }}$ was taken as $G_{\text {IIC }}$.

\subsection{Virtual crack closure technique}

\subsubsection{Theory}

The VCCT $[10,11]$ is based on the assumption that the energy released in extending a crack by a small amount, $\Delta a$, is equivalent to the work necessary to close the crack to its original length. In an FE analysis using the VCCT, the three strain energy release rate

Fig. 1. Double cantilever beam specimen. 
components $G_{I}, G_{I I}$ and $G_{I I I}$ are calculated at a crack front node by

$G_{I}=\frac{X^{\prime} \Delta u^{\prime}}{2 A} ; \quad G_{I I}=\frac{Y^{\prime} \Delta v^{\prime}}{2 A} ; \quad G_{I I I}=\frac{Z^{\prime} \Delta w^{\prime}}{2 A}$,

where $X^{\prime}, Y^{\prime}$ and $Z^{\prime}$ are the forces at nodes along the delamination front, $\Delta u^{\prime}, \Delta v^{\prime}$, and $\Delta w^{\prime}$ are the relative displacements of the node pairs ahead of each corresponding crack front node, and $A$ is the surface area created by crack growth. Eq. (3) requires the calculation of a local crack front coordinate system $\left(x^{\prime}, y^{\prime}, z^{\prime}\right)$ and modification to account for arbitrary element sizes. The VCCT is applicable for $2 \mathrm{D}$ or $3 \mathrm{D}$ analysis with linear and quadratic elements [11].

\subsubsection{VCCT in $\operatorname{Marc}^{T M}$}

The VCCT is implemented into $\operatorname{Marc}^{\mathrm{TM}}$ as a procedure for determining the strain energy release rate distribution at a crack front [13]. Crack front nodes are defined, and the solver determines the appropriate nodes, forces and areas to use for the crack growth calculation. This implementation follows the description given by Krueger [11], which accounts for a crack front of arbitrary shape.

Three main options are available for automatic crack front propagation: (1) remeshing; (2) propagation along element edges; (3) releasing constraints. In this work, the latter was used, as the other approaches were not available with 3D solid elements. For crack propagation by releasing constraints, two surfaces are connected using a bonded contact or multi-point constraints. In the current work, contact was used, which is similar to the approach implemented within Abaqus ${ }^{\circledR}$ [12].

At the end of every nonlinear analysis increment, the strain energy release rates are calculated using the VCCT. Crack growth onset is detected using Eq. (1), with either single-mode or mixedmode criteria for $G_{c}$. The crack is propagated by releasing the constraint at the crack front node, and is a critical step in ensuring that enough crack growth occurs within an increment. Restarting the increment after a single node release also enables any stress redistribution as a result of crack growth to be accounted for, as a new solution is determined for the structure following a small amount of crack growth. In this way, the analysis is not progressed until an appropriate amount of crack growth at a given load level has been determined, which allows for the incorporation of stress re-distribution, multiple crack paths and other non-linear effects. The restart approach also has important implications for the solver efficiency, as detailed further in the Discussion section.

Once propagation has been detected at a crack front, the solver locates the most appropriate node in the intact region to "grow" the crack. This is based on the definition of a crack growth direction. This direction can be aligned with the most critical mode, with the direction normal to the crack front, with a fixed user-defined vector, or determined with a criterion based on maximum principal stress [13]. In this work a fixed direction was defined, as this was suitable for the specimens and mesh configurations investigated.

\section{Finite element modeling}

The 2D and 3D FE models of the DCB and ENF specimens are shown in Figs. 3 and 4, which also illustrate the boundary conditions applied for these models. The models were based on those presented previously [16]. An additional ENF model with a modified mesh was generated, which is discussed further in the results section.

All models were divided into various sections with different mesh refinement along the specimen length. Although the dimensions of the elements varied, all meshes were based on a fine a

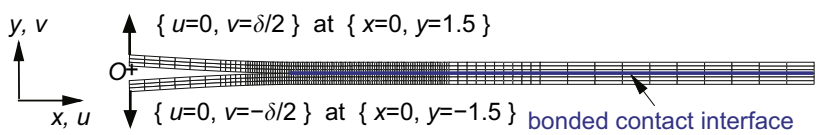

b

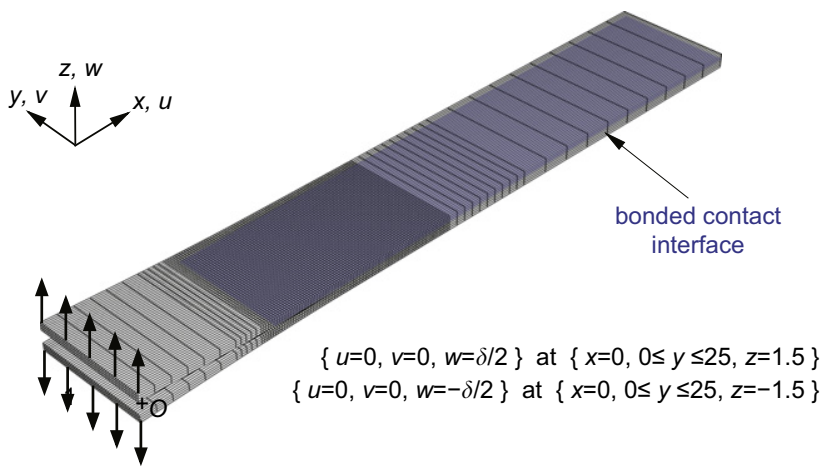

Fig. 3. DCB deformed specimen mesh with boundary conditions. (a) 2D model (b) 3D model.

a

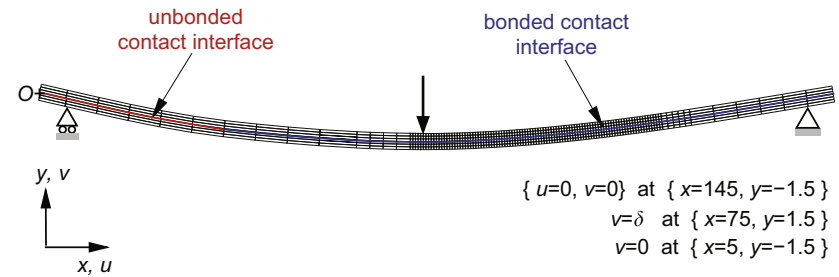

b

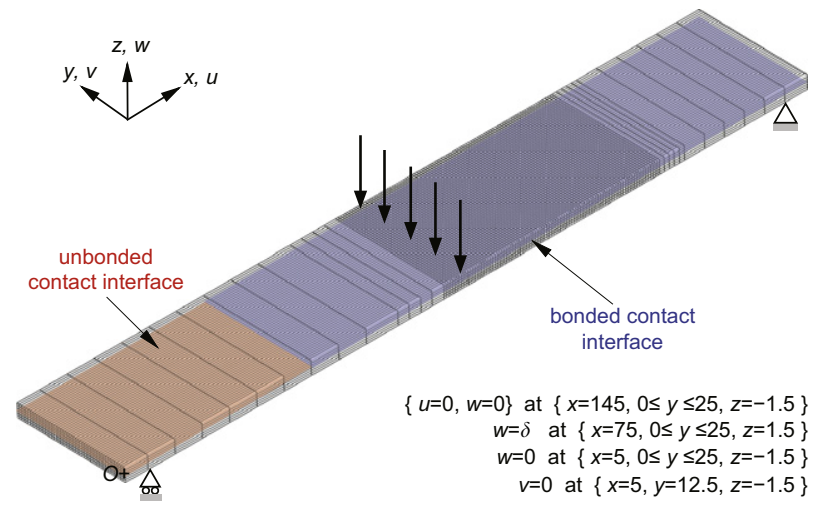

Fig. 4. ENF specimen mesh with boundary conditions. (a) 2D model, deformed (b) 3D model, undeformed.

mesh region that used elements of $0.5 \mathrm{~mm}$ length in the crack growth direction in the region immediately around the delamination front. This element length was selected in previous studies $[18,19]$, in which mesh convergence investigations were performed. All models used a uniform mesh across the width and thickness directions. Further detail of the modeling is given in Ref. [16].

For all models, the plane of delamination was modeled as a discrete discontinuity in the center of the specimen. To create the discrete discontinuity, each model was created from separate meshes for the upper and lower part of the specimens with identical nodal point coordinates in the plane of delamination. Two surfaces (top and bottom) were created on the meshes, and a bonded contact specified between them. 
All specimens were analyzed with 2D and 3D models. The 2D models used two-dimensional 4-node plane strain elements. The 3D models used 8-node reduced integration solid brick elements. The 2D and 3D models for a specimen used the same mesh scheme, as shown in Figs. 3 and 4. The nonlinear solver in Marc ${ }^{\mathrm{TM}}$ was used, which applied a full Newton-Raphson solution procedure with a load residual tolerance of 0.001. To minimize problems with numerical stability of the analysis caused by crack propagation, specimen loading was defined in terms of applied displacements. All models were run on a 32-bit Intel Core 2 Duo $2.25 \mathrm{GHz} \mathrm{CPU}$ processor.

\section{Analysis}

\subsection{Computation of strain energy release rates}

For each of the specimens, the computed strain energy release rate distribution across the delamination front was plotted versus the normalized specimen width, $y / B$, as shown in Figs. 5 and 6, for the 3D and 2D models. Distributions were calculated for a range of different delamination lengths, as part of the numerical benchmark results generation described in the next section. As strain energy release rate distributions for different delamination lengths were previously presented for the DCB specimen [16],

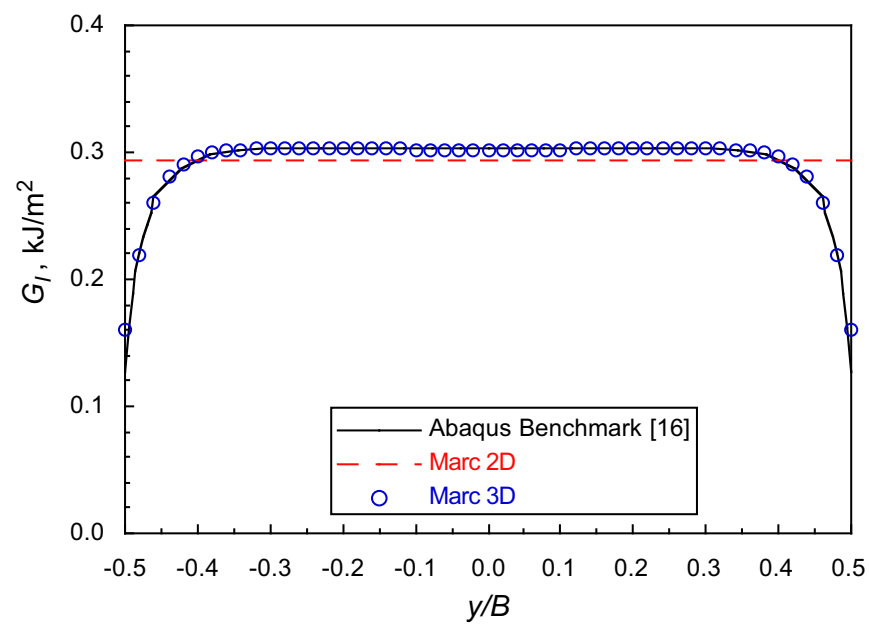

Fig. 5. Strain energy release rate distribution, DCB $(a=30.5 \mathrm{~mm}, \delta / 2=1.0 \mathrm{~mm})$.

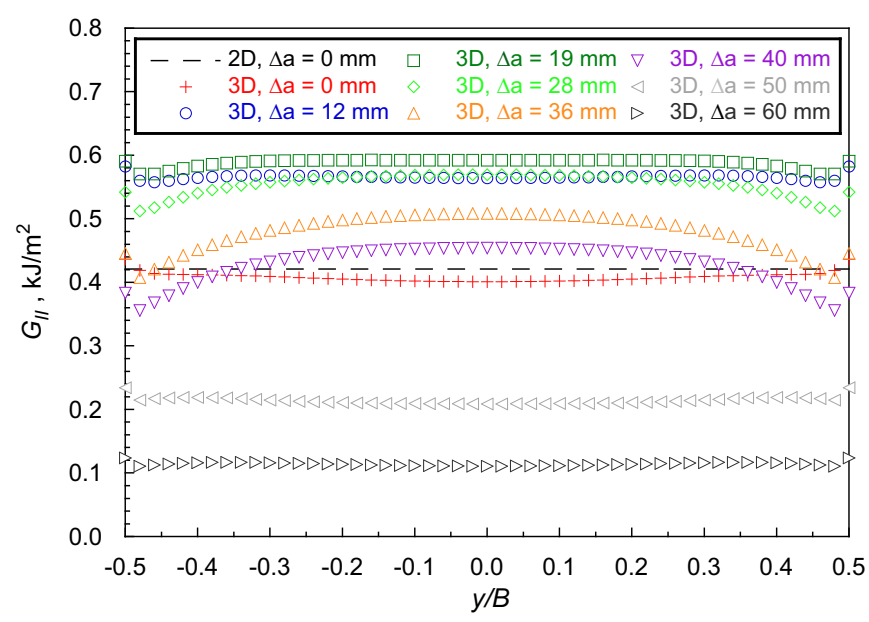

Fig. 6. Strain energy release rate distribution, $\operatorname{ENF}(\delta=4.0 \mathrm{~mm})$. the results in Fig. 5 are given for only one delamination length $(a=30.5 \mathrm{~mm})$ at an applied opening displacement $\delta / 2=1.0 \mathrm{~mm}$. The ENF results in Fig. 6 are given for all delamination lengths investigated for an applied center displacement $\delta=4.0 \mathrm{~mm}$. In Fig. 6, the delamination lengths are given as delamination growths $\Delta a$, relative to the baseline delamination length $a=30 \mathrm{~mm}$. For the DCB and ENF specimens, only the dominant mode component is shown (I and II respectively), as the others were negligible. For the DCB specimens, Fig. 5 demonstrates the excellent agreement between the results in $\operatorname{Marc}^{\mathrm{TM}}$ and those calculated using Abaqus ${ }^{\circledR}$ in previous work [16]. The results for all specimens demonstrate that 3D effects, such as anticlastic bending of the loaded specimen arms, cause the strain energy release rate distribution across the delamination front to be nonuniform, even for single-mode dominated specimens.

For the ENF specimen, the results in Fig. 6 show that for delamination growth up to $\Delta a=19 \mathrm{~mm}$, the average strain energy increased, and for delamination growth greater than $\Delta a=19 \mathrm{~mm}$ the average strain energy release rate decreased. This inflection point is associated with the change from unstable to stable crack growth. However, as the average strain energy release rate for delamination growth up to $\Delta a=40 \mathrm{~mm}$ was greater than the initial $(\Delta a=0 \mathrm{~mm}$ ) value, the onset of delamination would lead to unstable crack growth up to $\Delta a=40 \mathrm{~mm}$. The change to stable crack growth after $\Delta a=40 \mathrm{~mm}$ corresponds to the location of the central loading pin, as shown in Fig. 6. From the results in Fig. 6, it was found that the distribution of $G_{I I}$ across the delamination front changed slightly with delamination length. In this case, $G_{I I}$ peaked on the edges of the delamination front at shorter delamination lengths, and peaked in the center at longer delamination lengths. The distribution also became more curved at delamination lengths approaching the loading pin. The unstable nature of delamination growth in ENF specimens loaded quasi-statically, under displacement control, is well documented in the literature [20-22]. Although in experimental testing focused on material characterization, the delamination is not extended past this point, for numerical analysis this region remains valuable for benchmarking purposes.

\subsection{Creating benchmark delamination growth results}

The approach developed previously for generating numerical benchmark delamination growth results [16] was applied to the DCB and ENF specimens. For these specimens, a benchmark result set was extracted from a series of models with different delamination lengths. These models were not used to simulate delamination propagation, and were only used to obtain the load-displacement response and the strain energy release rate distribution for different delamination lengths. For each delamination length modeled, a failure index was calculated across the delamination front using Eq. (1), with the Reeder mixed-mode criterion and material data in Table 1 used to compute $G_{c}$ (Eq. (2)). Delamination growth onset was assumed when the failure index at the center of the specimen $(y / B=0)$ reached a value of unity. Specimen load-displacement response up to this point was also assumed to be linear. Subsequently, the displacement and load at delamination growth onset, $\delta_{\text {crit }}$ and $P_{\text {crit }}$, respectively, were computed by linearly scaling the prescribed displacement and load in each analysis ( $\delta$ and $P$ respectively), using the following relations [16]:

$\frac{G_{T}}{G_{c}}=\frac{P^{2}}{P_{c r i t}^{2}} \Rightarrow P_{c r i t}=P \sqrt{\frac{G_{c}}{G_{T}}}, \quad \delta_{c r i t}=\delta \sqrt{\frac{G_{c}}{G_{T}}}$,

The benchmark result set was constructed by plotting the displacement at delamination growth onset versus delamination length, as illustrated in Fig. 7 for the DCB specimen. This form is 


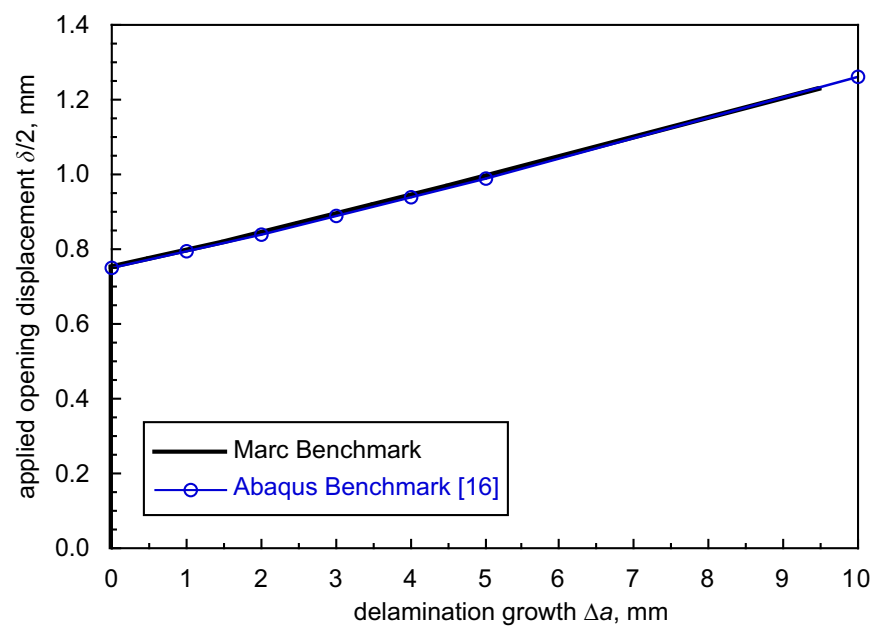

Fig. 7. DCB, benchmark delamination growth results.

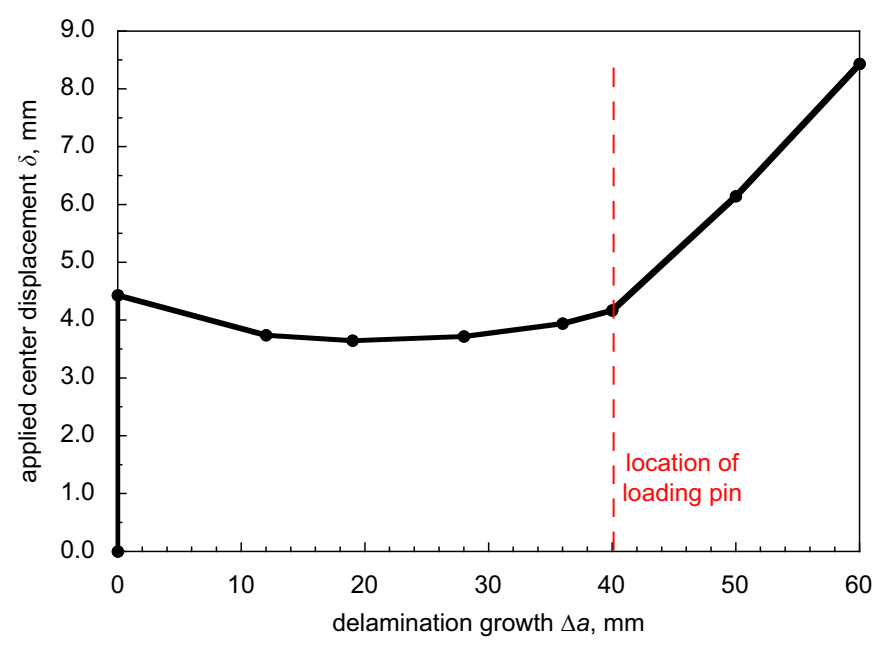

Fig. 8. ENF, Marc benchmark delamination growth results.

used for the benchmark result because all specimens were loaded via a prescribed displacement, and the change in delamination length is the most appropriate output for assessment of delamination growth. In previous work [16], the benchmark results were presented as load-displacement curves, to illustrate the application of Eq. (4).

The benchmark delamination growth results for the DCB and ENF specimens are presented in Figs. 7 and 8, respectively. Results from previous analyses with Abaqus ${ }^{\circledR}$ [16] are also included for the DCB specimen. The DCB results shows that the applied opening displacement increased with increasing delamination length, which indicates stable growth under displacement control. In contrast the ENF results indicated initial unstable delamination growth, as the critical displacement decreased with increasing delamination length. The comparison between the Abaqus $^{\circledR}$ and Marc ${ }^{\mathrm{TM}}$ results in Fig. 7 shows that the two solvers gave almost identical results for these models.

\subsection{Automated delamination propagation analysis: $D C B$}

For the DCB specimen, the results in Fig. 7 shows that delamination propagation was predicted to initiate at an applied opening displacement $(\delta / 2)$ of $0.75 \mathrm{~mm}$. Based on this, a two-step loading procedure was applied for the delamination propagation analysis, which involved using coarse time increments until just before failure, and fine increments for the region involving delamination propagation. Dividing the first step into relatively coarse time increments was possible as the load-displacement behavior of the specimen up to failure was expected to be linear. In the first step, a prescribed opening displacement of $\delta / 2=0.7 \mathrm{~mm}$ was applied in 10 increments. In the second step, the total prescribed displacement was increased to $\delta / 2=1.0 \mathrm{~mm}$. This was applied with a fixed time increment scheme of 50 increments $(\delta / 2=0.006 \mathrm{~mm}$ each increment).

In Marc $^{\mathrm{TM}}$, the solver has the capability to cut back the increment size in the event of convergence issues, and can activate damping when the time step is reduced below a defined minimum. Critically, no convergence issues were seen throughout any analyses, and damping was not required. This is quite different behavior from that seen previously with Abaqus ${ }^{\mathbb{R}}[16]$, where convergence issues associated with delamination growth caused significant cutbacks in the time increment and involved considerable computational expense. As a result, run times for delamination propagation analyses were within a minute for $2 \mathrm{D}$ models and generally within a few hours for 3D models, depending on the selection of increment size and amount of delamination growth. The efficiency of the solver is discussed further in a later section.

The results for the analysis of the DCB specimens are shown in Figs. 9 and 10. From the delamination growth results in Fig. 9, the VCCT technology gave very close comparison with the benchmark results, for both $2 \mathrm{D}$ and $3 \mathrm{D}$ models. The 3D models showed delamination growth at slightly lower applied displacements, which was due to the slightly higher strain energy release rates in the 3D models as shown in Fig. 5. From Fig. 10, the delamination was seen to propagate as a straight crack front, which contradicts the curved strain energy release rate distribution shown in Fig. 5. This propagation of a straight front is most likely related to the coarse element size in the width direction, and was also seen in previous analyses $[15,16]$.

The characteristic step pattern seen in the delamination growth results in Fig. 9 was caused by the step change in length as the delamination was propagated one element length at a time. The step sequence is caused by an initial increase in applied displacement until delamination growth, sudden nodal release, increase in displacement until next delamination growth, and repetition. These step changes in delamination length at a fixed applied displacement would produce a sequence of drops in the reaction load and the corresponding "saw-tooth" pattern in the load-displacement response [15,23]. The results also shows that the corners of the steps, just before a delamination growth event,

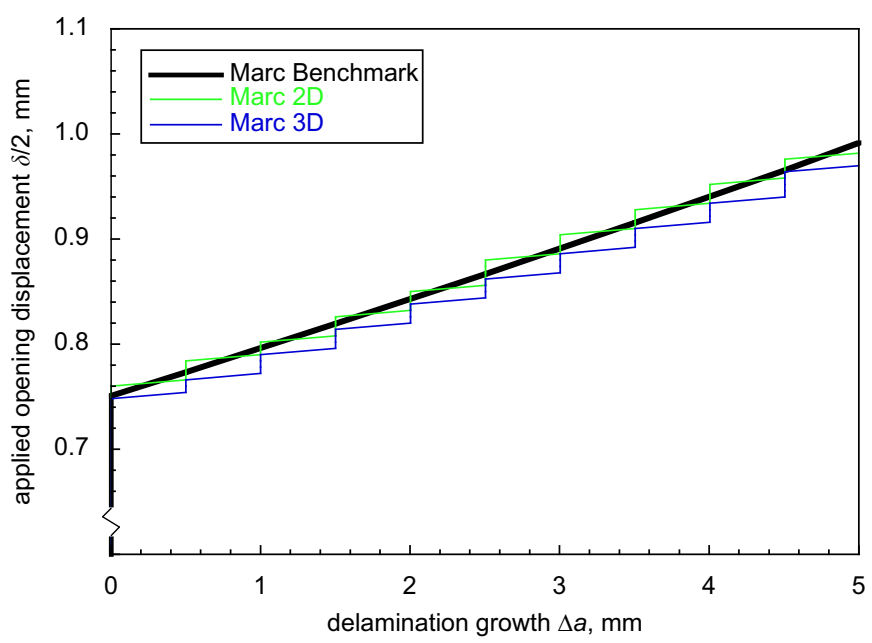

Fig. 9. DCB, applied displacement versus delamination growth. 


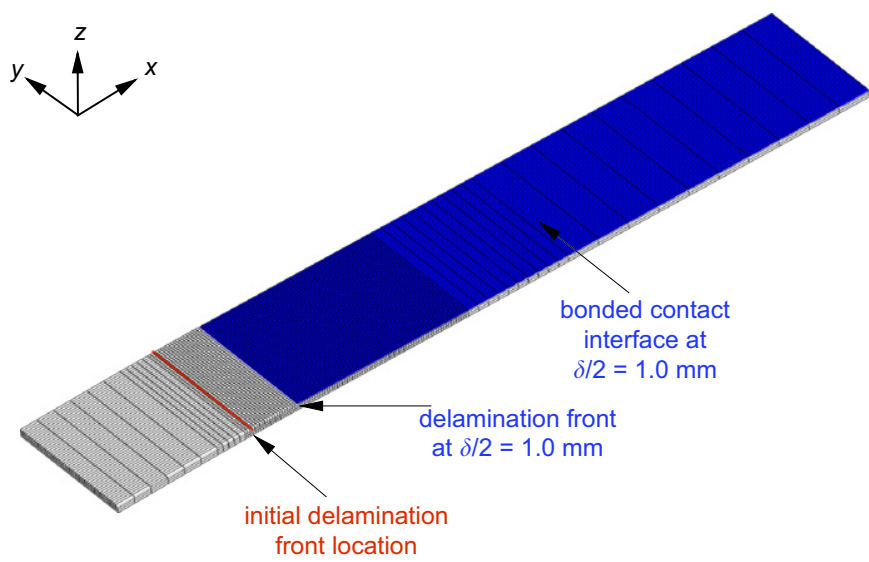

Fig. 10. DCB bottom sublaminate deformed mesh at $\delta / 2=1.0 \mathrm{~mm}$, with delamination interface.

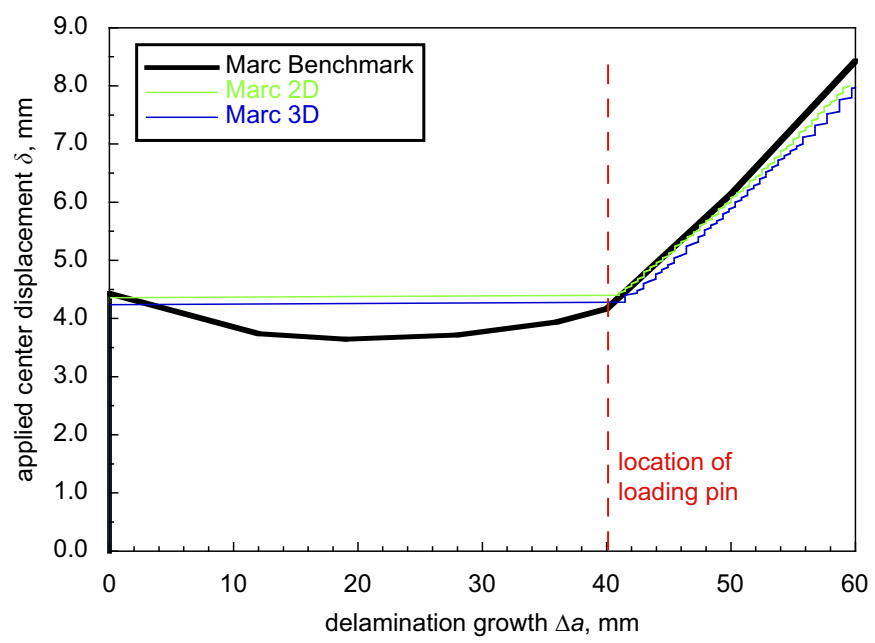

Fig. 11. ENF, applied displacement versus delamination growth.

provide the most suitable comparison with the benchmark results, which also allows coarse meshes to be adequately used.

\subsection{Automated delamination propagation analysis: ENF}

For the ENF model shown in Fig. 4, the analysis used two load steps with coarse and fine time step incrementation as discussed for the DCB specimen. In the first step, a prescribed center displacement of $\delta=4.0 \mathrm{~mm}$ was applied in 2 increments, while in the second step the total prescribed displacement was increased to $\delta=8.0 \mathrm{~mm}$ in 100 increments $(\delta=0.04 \mathrm{~mm}$ each increment). As with the DCB specimen, no significant convergence issues were seen and damping was not required.

The results for the analysis of the ENF specimens are shown in Figs. 11 and 12. As observed from these results, the VCCT technology was capable of accurately representing the benchmark delamination growth results for the baseline ENF mesh. As with the DCB specimen, delamination growth was initiated in the $3 \mathrm{D}$ model at a slightly lower applied displacement than the 2D model, which was due to a slightly higher strain energy release rate as shown in Fig. 6 for $\Delta a=0 \mathrm{~mm}$.

The results showed that the ENF specimen exhibited an initial period of large delamination growth. This was caused by the strain energy release rates remaining critical with increasing delamination length, as previously discussed. The first delamination growth event corresponded to a delamination growth step of $41 \mathrm{~mm}$, as shown in Fig. 11. This was followed by stable

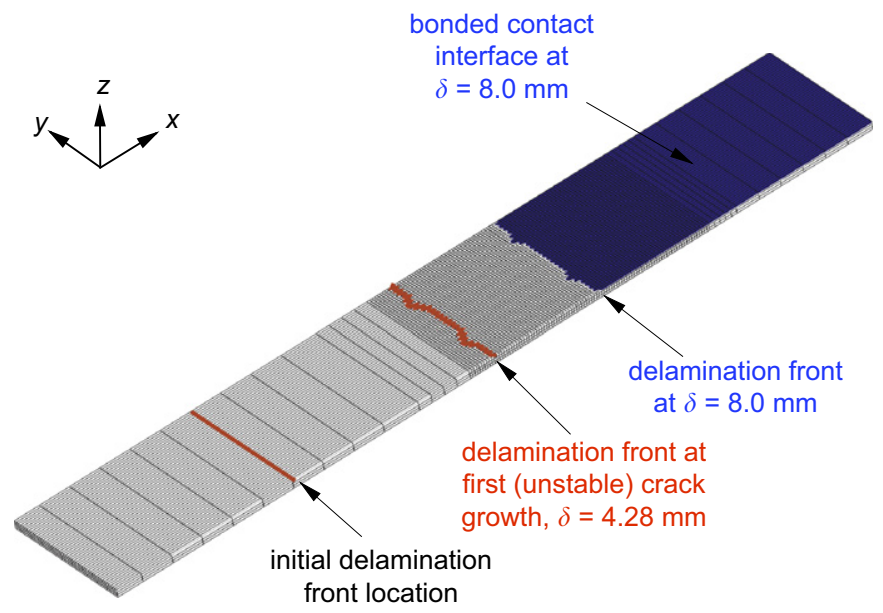

Fig. 12. ENF bottom sublaminate mesh with delamination front progression.

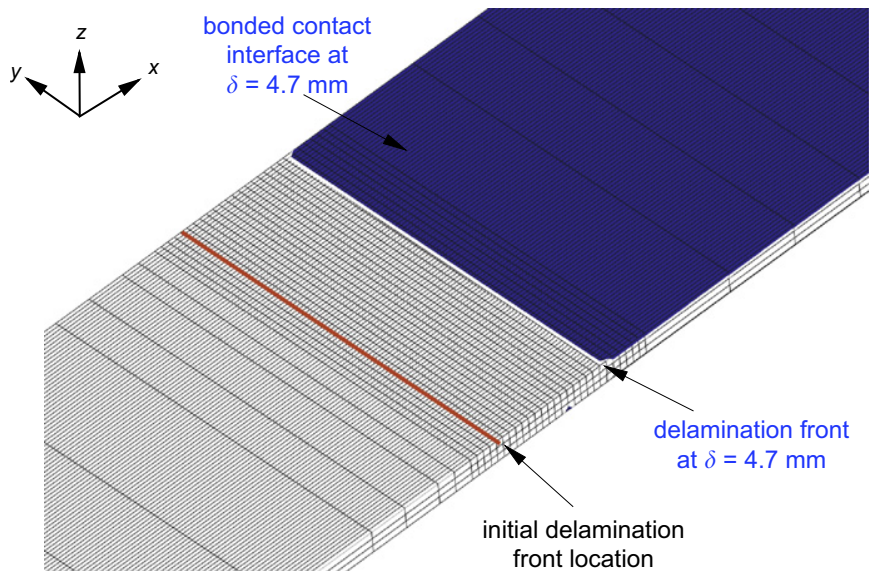

Fig. 13. ENF modified model bottom sublaminate mesh with delamination front progression.

delamination growth, where the benchmark and FE results correlated very closely.

From Fig. 12, the delamination front formed in the baseline mesh by the unstable delamination growth was jagged and nonuniform across the width. This irregular and unexpected pattern was considered a product of the delamination propagating through a mesh transition region, in addition to insufficient mesh density across the width. A modified mesh model was created to address these issues. Rather than using a large fine mesh region to capture all of the unstable delamination growth up to $41 \mathrm{~mm}$, it was decided to instead increase the length of the initial delamination up to an equivalent delamination growth of $35 \mathrm{~mm}$, and move the fine mesh region accordingly. This avoided the initial crack propagation through a mesh transition region. Additionally, the mesh density across the width was doubled, while for efficiency, the length of the fine mesh region was reduced. The ENF modified mesh can be seen in Fig. 13, which shows the fine and coarse mesh regions.

The results of the analysis of the ENF modified mesh model are shown in Fig. 13. The increased mesh density and initial delamination at $\Delta a=35 \mathrm{~mm}$ produced a straight delamination front without the jaggedness across the width. The delamination growth results for the models are shown in Fig. 14. From these results, the modified mesh gave closer comparison with the benchmark curve for crack growth through the fine mesh region. These results demonstrate that crack propagation through a mesh transition region could lead to an incorrect delamination front 


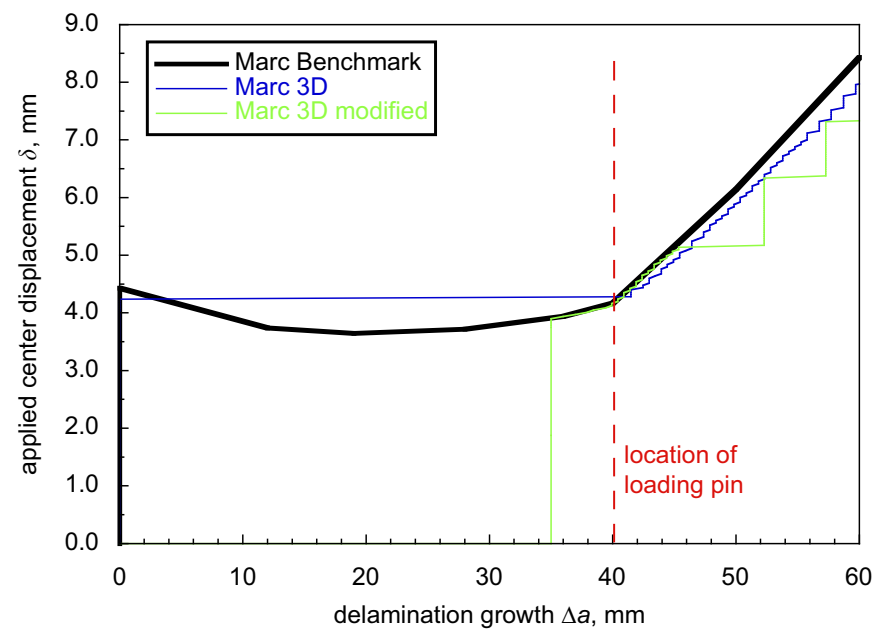

Fig. 14. ENF, applied displacement versus delamination length.

developing and propagating, and that the mesh refinement level was critical to accurately capturing the shape of the delamination front. The results in Fig. 14 for crack propagation through the coarse mesh region also clearly demonstrate the effect of large elements on the delamination growth, where the peaks of the large steps just prior to delamination growth gave the most suitable comparison with the benchmark results. This is discussed further in the following section.

\section{Discussion}

\subsection{Mesh transition}

At the transition between fine and coarse mesh regions, it was found that the Marc ${ }^{\mathrm{TM}}$ results showed a deviation from the expected behavior. As discussed previously for the DCB specimen, the peak displacements just prior to delamination propagation in each step give a consistent alignment with numerical benchmark results. The results at mesh transition regions suggest a likely omission in the VCCT implementation, caused by not accounting for different element lengths ahead and behind the delamination front. In the software documentation this aspect is not specifically addressed, though it is mentioned that a "regular" mesh is "advantageous for accuracy" [13].

As detailed in Refs. [10,11], the VCCT equations require a modification factor to account for uneven element lengths ahead and behind the crack front, such as those seen when the crack propagates through a mesh transition region. Although not clear in the above results, erroneous results were seen at mesh transition regions that were not evident elsewhere along the crack path. These erroneous results were seen also in Abaqus ${ }^{\circledR}$ results in Ref. [24], and suggest a similar implementation omission in both codes.

To further illustrate the effect of mesh transitions, a 2D DCB model with several mesh transition regions was analyzed, with the mesh and the results shown in Fig. 15. The erroneous results were most clear for the transition to smaller elements, as is shown at $\Delta a=5.25 \mathrm{~mm}$, where an under-estimation of the strain energy release rates due to the mesh transition prevented delamination growth from occurring and following the benchmark results. The same difference is less evident though still occurring in the two transitions to larger meshes at $\Delta a=1.0 \mathrm{~mm}$ and $\Delta a=7.25 \mathrm{~mm}$. For the 2D DCB model in this example the errors did not propagate, so that predictions in a regular mesh following a mesh transition region returned to follow the

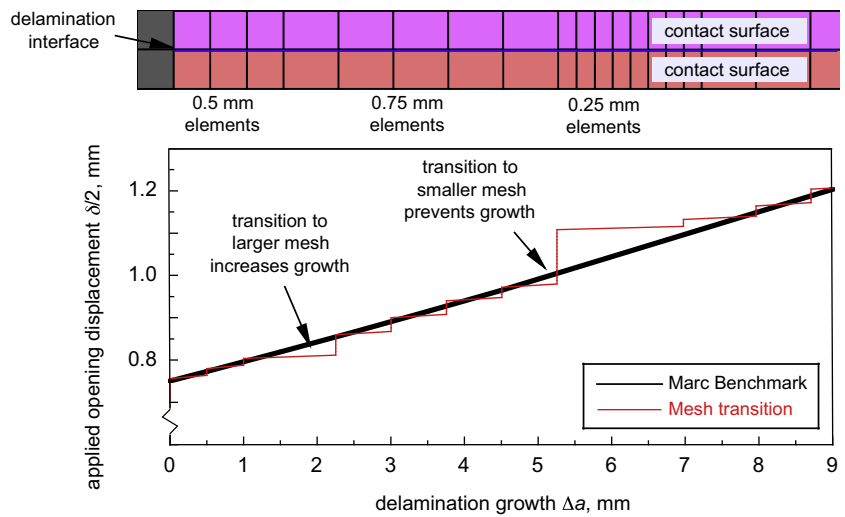

Fig. 15. Effect of mesh transition regions, DCB 2D.

benchmark solution. However, the results for the 3D ENF model demonstrated that an incorrect delamination front could develop due to a mesh transition region and be propagated.

\subsection{Solver comparison: $\operatorname{Marc}^{T M}$ and Abaqus $^{\circledR}$}

A comparison of results from the Marc ${ }^{\mathrm{TM}}$ analysis with previous Abaqus ${ }^{\circledR}$ results [16] indicated a difference in the solution process between the two implementations. Critically, in the Marc $^{\mathrm{TM}}$ implementation of VCCT [13], once crack growth is detected in an increment the crack front node is released and the increment is restarted. This allows for multiple crack growth instances to occur in one increment, and allows for coarse time increments to be used, which is computationally efficient. By comparison in the Abaqus ${ }^{\circledR}$ implementation [12], only one crack growth instance can occur in each increment, so it is necessary to reduce the increment size considerably in order to ensure that no overestimation occurs. The failure index determined from the strain energy release rates is monitored, and the user defines a limit on the amount that the failure index can exceed a value of 1.0. This overshoot limit, or "release tolerance", as such becomes another parameter in the model that requires careful selection [16]. So, while the Abaqus ${ }^{\circledR}$ approach decreases the increment size to suit the crack growth, in Marc ${ }^{\mathrm{TM}}$ the crack growth is increased to suit the increment size, so that larger increments can be used with increased computational efficiency.

Another important difference between Marc $^{\mathrm{TM}}$ and Abaqus ${ }^{\circledR}$ was the convergence difficulties and the subsequent damping required. In previous work using Abaqus ${ }^{\circledR}$ [16], it was found that damping, or "stabilization", needed to be added to the solver, in order to get a solution in light of the convergence issues. The introduction of stabilization parameters required considerable effort in parametric investigation in order to determine a suitable compromise between damping and solution accuracy. In contrast, for the models considered in this work with Marc ${ }^{\mathrm{TM}}$, no severe convergence issues were recorded and damping was not applied in any of the solutions.

The two aspects of differing time increment requirements (caused by allowing multiple crack growth events in an increment) and the requirement for damping meant that the Marc ${ }^{\mathrm{TM}}$ solver was considerably more computationally efficient than the Abaqus ${ }^{\mathbb{R}}$ solver for the models analyzed. This was most noticeable for the 3D models, where for example the Abaqus ${ }^{\circledR}$ solver required run times of up to several days [16], while the Marc ${ }^{\mathrm{TM}}$ solver experienced run times of only several hours. The Abaqus analyses were run on a different machine (Dual-Core AMD Opteron $^{\mathrm{TM}}$ Processor $8220 \mathrm{SE}$ ), so that a true quantitative comparison could not be made. Further, the convergence criterion for residuals was different for the two solvers, where the Marc ${ }^{\mathrm{TM}}$ 
analyses used a maximum residual of 0.001 , whereas the Abaqus ${ }^{\circledR}$ analysis used the default setting of 0.005 . However, the comparison clearly highlights the considerable difference between the two solvers, particularly as the machine for the Abaqus analyses had a superior processor. The time difference between the solvers is further exacerbated by the introduction of the release tolerance and stabilization parameters, which typically require parametric investigations to determine.

\subsection{Further work}

The investigation in this paper assessed the static delamination growth capabilities of Marc ${ }^{\mathrm{TM}}$ for single mode I and II specimens. Although the scope of the current work was limited to an assessment of single mode delamination growth, demonstration of the numerical benchmarking approach, and comparison with previous results in Abaqus ${ }^{\circledR}$, a more comprehensive assessment of static delamination growth capabilities would consider:

- Mixed-mode specimens, which could involve the MMB specimen for mixed mode I-II [5], or configurations involving circular or elliptical delaminations [25]. Analysis of the SLB specimen involving mixed-mode $I / I I\left(G_{I I} / G_{T}=0.4\right)$ delamination growth was presented in previous work $[16,17]$.

- Delamination growth through irregular 3D meshes, particularly where the delamination front is not parallel to the element edge. This type of delamination growth has been studied by other authors [26,27], and shown to present considerable challenges for the determination of suitable strain energy release rates and implementation of automated delamination growth algorithms.

- Specimens where the re-distribution of stresses following delamination growth is important for accurate analysis. This would include specimens involving multiple delaminations [28], buckling-driven delamination growth [29,30] and crack kinking [31].

- Comparison with experimental results for some or all of the benchmark cases. Although all experimental results present challenges as previously discussed, an assessment on the capabilities of a code to capture real life behavior must include a comparison with experimental results, as demonstrated in previous publications $[17,23,24]$.

As such, the results and conclusions generated in this work are limited to only the specimens and mesh configurations investigated. It is expected that the capability of the software codes to represent benchmark data would be further challenged in the above cases, particularly those involving delamination growth with an irregular 3D mesh. However, for any configuration, the application of a numerical benchmarking approach would be easily achievable, and would offer the ability to assess the capabilities of the software codes without the issues associated with experimental or analytical benchmarks.

\section{Summary and conclusions}

The delamination propagation simulation capabilities of the commercial FE code Marc ${ }^{\mathrm{TM}} 2008 \mathrm{r} 1$ with the VCCT was assessed. Benchmark delamination growth results for DCB and ENF specimens were generated using a previously developed numerical approach [16]. Specimens were analyzed using 3D and 2D models, and compared to previous analyses using Abaqus ${ }^{\circledR}$ with the VCCT implemented. The key conclusions of the work were:

- The approach applied in this work for using a numerical benchmark is a practical and efficient comparative technique, and offers advantages over experimental and analytical benchmarks as it involves a direct comparison between identical models.

- The VCCT implementation in Marc ${ }^{\mathrm{TM}}$ was capable of replicating the benchmark data for the DCB and ENF specimens, in terms of delamination growth behavior.

- No issues were seen with convergence during the Marc ${ }^{\mathrm{TM}}$ analyses, so that no damping was applied, and in general, the analyses were not considered highly sensitive to the solver input parameters.

- The capacity in Marc ${ }^{\mathrm{TM}}$ to automatically internally restart the increment after crack growth, and hence capture multiple crack growth events in one increment, was highly beneficial in terms of computational efficiency.

- Based on the previous two points, the analyses in Marc ${ }^{\mathrm{TM}}$ were considerably more computationally efficient than those previously conducted in Abaqus ${ }^{\mathbb{R}}$. Problems involving identical meshes across all codes took several hours in Marc ${ }^{\mathrm{TM}}$, and several days in Abaqus ${ }^{\mathbb{R}}$, although these were run on different machines.

- The implementation of the VCCT in Marc ${ }^{\mathrm{TM}}$ did not appear to account for irregular mesh lengths ahead and behind the crack front, and the use of mesh transition and fine mesh regions needed to be managed carefully in order to ensure an appropriate delamination front was formed.

Overall, it is clear that delamination propagation modeling is rapidly evolving in commercial FE codes, with each new software release involving new technologies and developments. This highlights the need for benchmarking techniques that are capable of isolating and assessing the key requirements for delamination propagation simulation. This need is clearly demonstrated by the results in this work, where significant differences were seen between two commercial codes implementing nominally the same technique.

\section{Acknowledgments}

This material is based on the work supported by National Aeronautics and Space Administration, Langley Research Center under Research Cooperative Agreement no. NNL09AA00A awarded to the National Institute of Aerospace. The research was supported by the Aircraft Aging and Durability Project as part of NASA's Aviation Safety Program, and an RMIT University Research Leave Award. The analyses were performed at the Durability, Damage Tolerance and Reliability Branch at NASA Langley Research Center, Hampton, Virginia, USA. The authors would like to thank Per Nordlund of MSC.Software Corporation for his support and advice.

\section{References}

[1] T.K. O'Brien Characterization of Delamination Onset and Growth in a Composite Laminate. Damage in Composite Materials, ASTM STP 775: American Society for Testing and Materials, 1982, pp. 140-67.

[2] T.E. Tay, Characterization and analysis of delamination fracture in composites-an overview of developments from 1990 to 2001, Appl. Mech. Rev. 56 (1) (2003) 1-32.

[3] T.K. O'Brien, Fracture mechanics of composite delamination, in: ASM Handbook, vol. 21, Composites: ASM International, 2001, pp. 241-245.

[4] ASTM D 5528-94a, Standard Test Method for Mode I Interlaminar Fracture Toughness of Unidirectional Fiber-Reinforced Polymer Matrix Composites. Annual Book of ASTM Standards: American Society for Testing and Materials; 2000.

[5] ASTM D 6671-01, Standard Test Method for Mixed Mode I-Mode II Interlaminar Fracture Toughness of Unidirectional Fiber Reinforced Polymer Matrix Composites. Annual Book of ASTM Standards: American Society for Testing and Materials; 2000.

[6] A.J. Russell, K.N. Street, Factors affecting the interlaminar fracture energy of graphite/epoxy laminates, in: T. Hayashi, K. Kawata, S. Umekawa (Eds.), 
Progress in Science and Engineering of Composites, ICCM-IV, Tokyo, 1982, pp. 279-286.

[7] T.K. O’Brien, Composite Interlaminar Shear Fracture Toughness, GIIc: Shear Measurement or Sheer Myth? Composite Materials: fatigue and Fracture, Seventh Volume, ASTM STP 1330: American Society for Testing and Materials; 1998 , pp. 3-18.

[8] M.L. Benzeggagh, M. Kenane, Measurement of mixed-mode delamination fracture toughness of unidirectional glass/epoxy composites with mixedmode bending apparatus, Compos. Sci. Technol. 56 (4) (1996) 439-449.

[9] J. Reeder, 3D Mixed-Mode Delamination Fracture Criteria-An Experimentalist's Perspective, American Society for Composites, 21st Annual Technical Conference, Dearborn, MI, USA, 17-20 September, 2006.

[10] E.F. Rybicki, M.F.A. Kanninen, Finite element calculation of stress intensity factors by a modified crack closure integral, Eng. Fracture Mech. 9 (1977) 931-938.

[11] R. Krueger, Virtual crack closure technique: history, approach and applications, Appl. Mech. Rev. 57 (2) (2004) 109-143.

[12] Abaqus ${ }^{\mathbb{R}} 6.8$ Documentation. Providence, RI, USA: Dassault Systèmes Simulia Corporation; 2008.

[13] Marc $^{\mathrm{TM}}$ and Mentat ${ }^{\mathrm{TM}} 2008$ r1 User Manuals. Santa Ana, CA, USA: MSC.Software Corporation; 2008.

[14] D. Xie, S.B. Biggers Jr, Progressive crack growth analysis using interface element based on the virtual crack closure technique, Finite Elements Anal. Design 42 (2006) 977-984.

[15] A.C. Orifici, R.S. Thomson, R. Degenhardt, C. Bisagni, J. Bayandor, Development of a finite-element methodology for the propagation of delaminations in composite structures, Mech. Compos. Mat. 43 (1) (2007) 9-28.

[16] R. Krueger An Approach to Assess Delamination Propagation Simulation Capabilities in Commercial Finite Element Codes. NASA/TM-2008-215123, National Aeronautics and Space Administration, Hampton, VA, USA; 2008.

[17] A.C. Orifici, R. Krueger Assessment of Static Delamination Propagation Capabilities in Commercial Finite Element Codes Using Benchmark Analysis. NASA/CR-2010-216709, National Aeronautics and Space Administration, Hampton, VA, USA; 2010.

[18] R. Krüger, Three Dimensional Finite Element Analysis of Multidirectional Composite DCB, SLB and ENF Specimens, Institute for Statics and Dynamics of Aerospace Structures, University of Stuttgart, 1994.

[19] R. Krueger, D. Goetze, Influence of Finite Element Software on Energy Release Rates Computed Using the Virtual Crack Closure Technique, NIA Report No. 2006-06, NASA/CR-2006-214523, 2006.
[20] L.A. Carlsson, J.W. Gillespie, R.B. Pipes, On the analysis and design of the end notched flexure (ENF) specimen for mode II testing. J. Compos. Mater. 20 (1986) 594-604.

[21] P. Davies, B.R.K. Blackman, A.J. Brunner, Standard test method for delamination resistance of composite materials: current status, Appl. Compos. Mater. 5 (1998) 345-364.

[22] T.K. O'Brien, G.B. Murri, S.A. Salpaekar Interlaminar Shear Fracture Toughness and Fatigue Thresholds for Composite Materials. In: Lagace PA, editor. Composite Materials: fatigue and Fracture, Second Volume ASTM STP 1012: American Society for Testing and Materials; 1989, pp. 222-250.

[23] A.C. Orifici, R.S. Thomson, R. Degenhardt, S. Büsing, J. Bayandor, Development of a Finite Element Methodology for Modelling Mixed-Mode Delamination Growth in Composite Structures, 12th Australian International Aerospace Congress, Melbourne, Australia, 19-22 March 2007.

[24] R. Krueger, Assessment of Delamination Propagation Capabilities in Commercial Finite Element Codes, ASC 23rd Technical Conference on Composite Materials, Memphis, TN, USA, 9-11 September 2008.

[25] Y. Murakami, Stress intensity factors handbook, Pergamon, New York, 1987

[26] D. Xie, S.B. Biggers Jr, Strain energy release rate calculation for a moving delamination front of arbitrary shape based on the virtual crack closure technique. Part I: formulation and validation, Eng. Fracture Mech. 73 (2006) $771-785$.

[27] D. Xie, S.B. Biggers Jr, Strain energy release rate calculation for a moving delamination front of arbitrary shape based on the virtual crack closure technique. Part II: sensitivity study on modeling details, Eng. Fracture Mech. 73 (2006) 786-801.

[28] G.C. Alfano, M.A., Finite element interface models for the delamination analysis of laminated composites: mechanical and computational issues, Numerical Met. Eng. 50 (2001) 1701-1736.

[29] J.D. Whitcomb, Analysis of a laminate with a postbuckled embedded delamination, including contact effects, J. Compos. Mater. 26 (1992) 1522-1535.

[30] J. Reeder, K. Song, P. Chunchu, D. Ambur, Postbuckling and growth of delaminations in composite plates subjected to axial compression, 43rd AIAA/ASME/ASCE/AHS/ASC Structures, Structural Dynamics and Materials Conference, Denver, CO, USA, 22-25 April 2002.

[31] D. Xie, A.M. Waas, K.W. Shahwan, J.A. Schroeder, R.G. Boeman, Computation of energy release rates for kinking cracks based on virtual crack closure technique, Comput. Modeling Eng. Sci. 6 (2004) 515-524. 